\title{
A study on the development of joint tracking-based exercise contents technology for improving the strength of older people
}

\author{
Donggyu Choi, Minyoung Kim, Jongwook Jang \\ Department of Computer Engineering, Dong-Eui University, Korea
}

\begin{tabular}{l}
\hline \hline Article Info \\
\hline Article history: \\
Received Jul 31, 2020 \\
Revised Dec 11, 2020 \\
Accepted Dec 24, 2020 \\
\hline
\end{tabular}

Keywords:

Calculation

Computer vision

Exercise contents

Machine learning

Older people

\begin{abstract}
Currently, Korea's population is aging rapidly, and there is a lot of interest in the area of life in old age. Especially, as you get older, your ability to exercise gradually decreases, and you need to exercise continuously for your health. As a result, Korea older people exercise more than welfare powerhouse Japan. However, recently studies show that physical function lags even further. The results are based on a lack of diversity in motion. In Korea, people enjoy walking, hiking, and riding bicycles, but in Japan, there is a difference in the quality of exercise such as muscle-building exercise class, ball exercise, and underwater exercise. The old Korean ways of exercising are all high-intensity sports, but some do not know whether the purpose is for social club purposes. In this paper, real-time joint tracking using a single camera and deep learning was carried out to study the development direction of content that can measure or improve exercise ability to improve actual muscle strength.
\end{abstract}

This is an open access article under the CC BY-SA license.

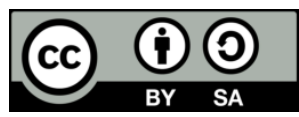

\section{Corresponding Author:}

Jong-Wook Jang

Department of Computer Engineering

Dong-Eui University

176, Eomgwang-ro, Busanjin-gu, Busan, Republic of Korea

Email: jwjang@deu.ac.kr

\section{INTRODUCTION}

Currently, the quality of life has improved significantly with income growth, but population aging is accelerating due to advanced countries. In addition, welfare and various health measures are changing very quickly [1-4]. Older people in Korea usually choose to go hiking for exercise. Because there are many mountains due to the characteristics of the topography, it can be the simplest. But contrary to what you think, mountaineering in older people can be more dangerous to the joints. Actual research shows that Japan has more exercise than older people, but has less practical effect. This is not because climbing is done properly, but because it is mostly a related gathering and lacks diversity as an exercise [5].

Hospitals believe that this phenomenon has a significant impact on the rehabilitation of patients, with a small lack of exercise. Usually, older people are very short of exercise due to various circumstances. However, older people who are easily hurt by their nature will be used to lying down because exercise is not already a habit even if they become patients and are hospitalized later. In fact, exercise is known to be very effective not only in rehabilitation but also in prevention, so it should continue even if it is not a situation to be done [6-9].

In particular, the exercise rate of modern people, which had previously been lacking due to the coronavirus, has been decreasing recently. People who used to live mostly at home finally felt the seriousness of the situation, and people came up with a number of ways to solve this situation. The first was to sell online real-time streaming and exercise equipment to make it possible at home. In addition to that, Vlog-style yoga, 
Pilates, and fitness were uploaded briefly through YouTube so that various people could try it [10]. However, there is no way to give feedback in this way. Also, it is not easy for older people to access Internet content. It is not a proper exercise because there are no conditions for performance, such as whether or not they are doing the correct action or counting the number of times [11-13].

In this paper, the necessity and direction of developing contents for the body of old age, which can be easily checked indoors, were studied in order to balance these exercises. At the same time, it showed the angular computation of the joints used inside the content and their utilization. Users can run and exercise at any time with a camera and a computer capable of running the program, and hospitals can use data and conduct research through it. This paper is an extension of work originally presented in Korea Institute of Information and Communication Engineering [14].

\section{RESEARCH METHOD}

The direction of the study is: First of all, we use joint tracking algorithms of frequently used commercial models [15-17]. The first step for customizing is to evaluate the joint position using the average of each frame to improve accuracy. The result is then produced by calculating the angle of the line connecting the joint and the joint through vector operation. This is a plane angle, so it can be represented in 3D form by additional operations.

\subsection{Joint position correction}

An algorithm to track joints is first required before carrying out the proposed method. From OpenNI2 to deep learning, there are a variety of ways. When one of the algorithms is selected and used, the location of the joint can be easily identified. However, joint tracking may not be smooth due to the color of the background and the filming environment. Although the problem cannot be prevented in the first place, there is a way to solve the problem caused by a momentary error.

The first solution to the error is to use machine learning. Area data is collected for about 3 to 5 seconds because it cannot be performed with a few locations of the first recorded joints. Because of the more sensitive issues in which the collected area data stays longer, if used immediately, the data will continue to focus in similar locations. In addition, the task to handle clustering consumes the task and therefore wastes resources. The error solution used in this paper is the average location operation by frame. Just like using machine learning, this method requires data collection in the early stages. However, apart from the actual real-time data, the actual location and the position of the joint are corrected for the average data. If sensitive responses to nearby values, which are the advantages of clustering, show a slight difference, other data will be output at the desired value. Among the representative clustering, the formula for K-means algorithm and the figure shown using it are shown in Figure $1[18,19]$.
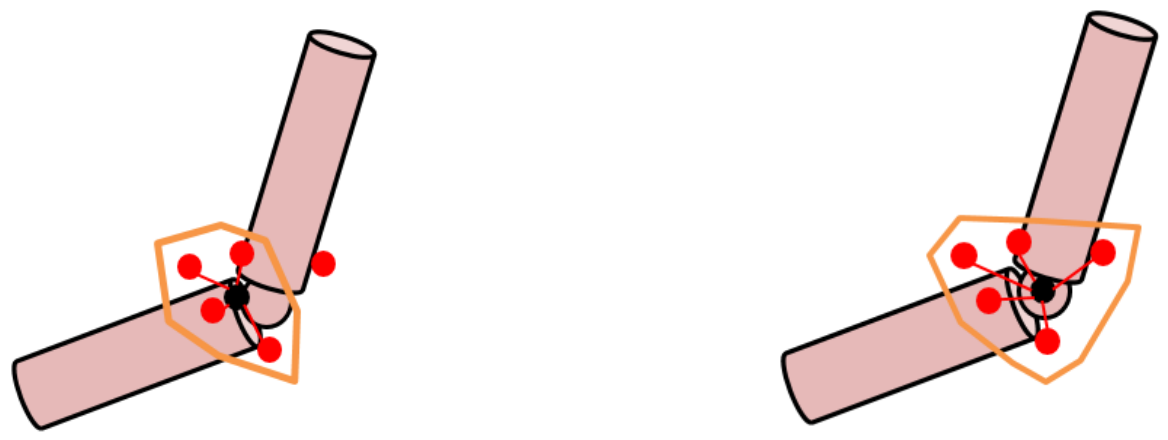

Figure 1. Results image according to data processing

Figure 1 is shows data from joint tracking using K-means clustering for images on the left. This results in outputting different data from more desired values when the nearby object values are skewed to one side for the center point you want to find. On the other hand, it can be seen that a more sensitive response to average data results in the output of central data from the entire data.

$$
\arg _{s} \min \sum_{i=1}^{k} \sum_{x \in S_{i}}\left\|x-\mu_{i}\right\|^{2}
$$




\subsection{Joint angle calculation}

The angular operation of the joint will take place in two directions. This is divided into when only one camera is used and when there is depth data. When only one camera is used, there is a problem that only computes the data that appears in the cross-sectional area because the angular computation consists of the data in the plane. However, the presence of depth data can produce more significant results than the angular data on the plane because the angle of the joint can be recognized in three dimensions. In fact, a person's body does not move in two dimensions, but in three dimensions. Figure 2 shows the two-dimensional and three-dimensional angles.

Figure 2 shows the angle of the joint that can be measured according to the performance of the camera. The image onthe left shows the angle that can be calculated with one general camera, and the image on the right shows the three-dimensional angle that can be calculated through the depth camera or calibration of two or more cameras. In Figure 3, the position according to the angle calculation described in Figure 2 is shown through the $\mathrm{x}, \mathrm{y}$, and $\mathrm{z}$ axes. In the case of 'angle2Dx', it represents the angle of the section including the $\mathrm{x}$ and $\mathrm{y}$ axes, 'angle2Dy' represents the angle of the cross section including the $\mathrm{x}$ and $\mathrm{z}$ axes.
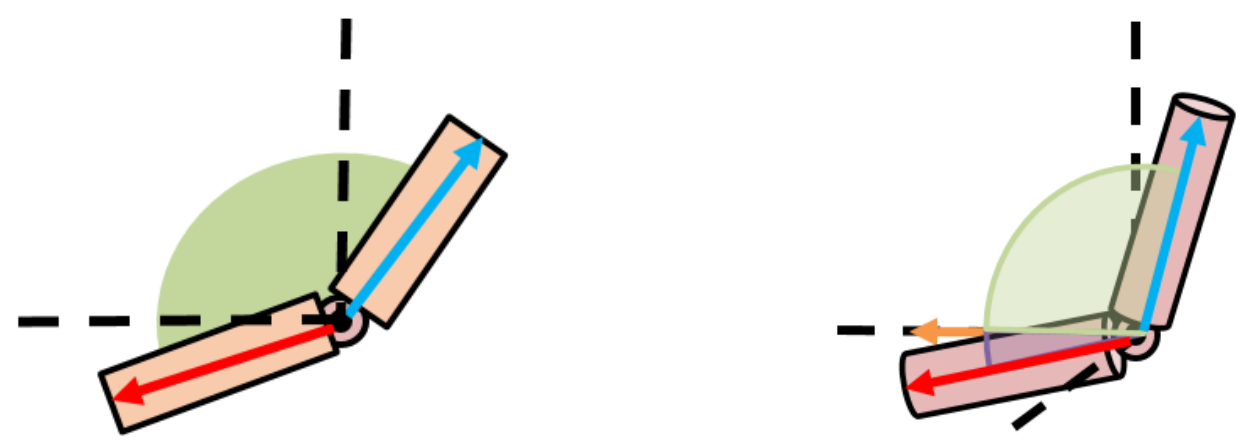

Figure 2. Comparison of joint data in two and three dimensions

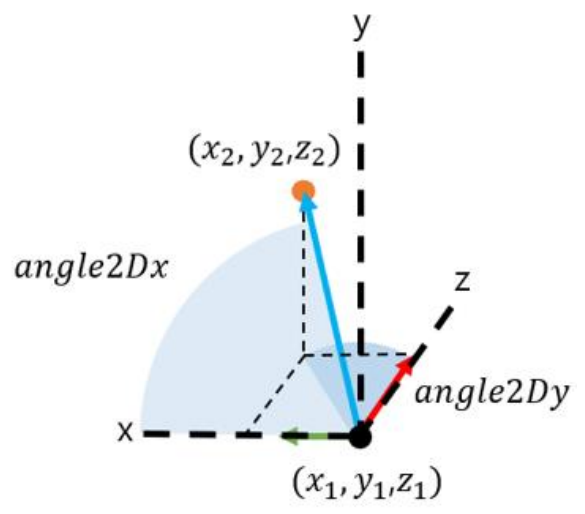

Figure 3. Graph for articulation angle computation

The formulas and methods for computing the image of the above joint are as (2), (3), and (4).

$$
\begin{aligned}
& x_{1}-x_{2}=X, y_{1}-y_{2}=Y, z_{1}-z_{2}=Z \\
& P_{x}=(\mathrm{X}, \mathrm{Y}), P_{y}=(\mathrm{X}, \mathrm{Z}) \\
& \text { angle } 2 D x=\arctan P_{x} \div(180 \pi), \text { angle } 2 D y=\arctan P_{y} \div(180 \pi)
\end{aligned}
$$

The overall formula follows the angular computation of the two points of the vector [20, 21]. Compute the error values for the two joint measurement positions in the formula of (2). Then, take the X, Y, and $\mathrm{Z}$ axes as shown in (3) and perform an operation to find the angles of two points in the vector by forming 
an angle of two cross sections in the formula of (4). This is to measure the angle on the original plane. However, if this is performed on two plane sections, the angle of the front section and the side section of the side can be determined. Therefore, it is possible to check the angle of depth as well as the angle of the existing section.

\subsection{Exercise movements for the elderly}

Exercise for the elderly consists of exercises that maintain or endure posture so that it is easy to maintain balance in the body. The use of arms in existing movements has been added. Figure 4 shows an image of a puppet of what to do. The name of the two moves is one with one leg raised, both arms raised (similar to the warrior pose of yoga) and arms wide open. Motion was added in contact with the essentials to achieve balance through various papers. Since balance requires the strength of the lower body, the movements in Figure 4 are mainly lower body movements.
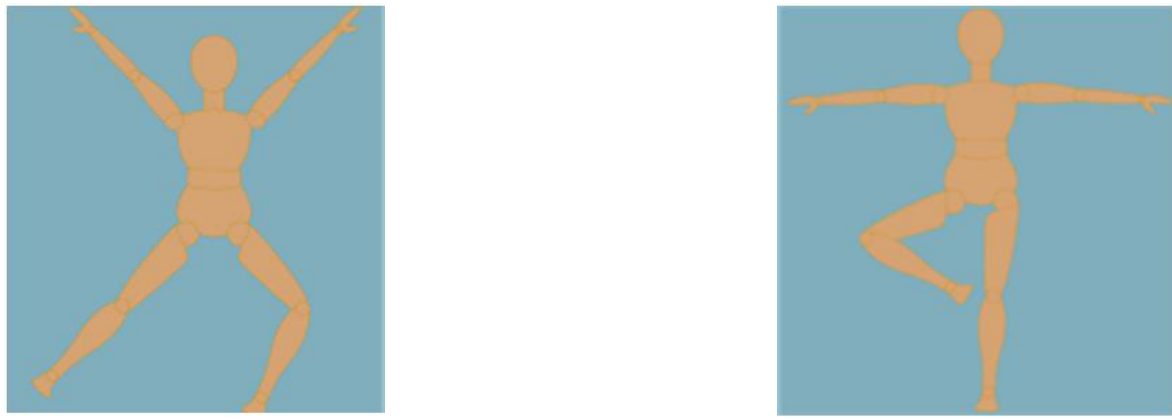

Figure 4. Image of holding one foot and balancing (left), warrior posture (right)

\section{RESULTS AND DISCUSSION}

Many studies are currently under way on joint tracking or data processing with two-dimensional cameras [22-24]. Therefore, three-dimensional cameras were used for implementation, but basic operations will be performed in two-dimensional camera images to achieve accurate results. For the aforementioned three dimensions, the function of this program can be expanded if the three-dimensional angle can be identified using either the Depth camera or two 2-dimensional cameras with calibration. Figure 5 shows the program contents of the research results that help the elderly to exercise simply. According to the actual motion, the puppet moves at the same angle as the joint. Based on the aforementioned algorithm, we selected important joints and performed the simple motions described earlier [25, 26].

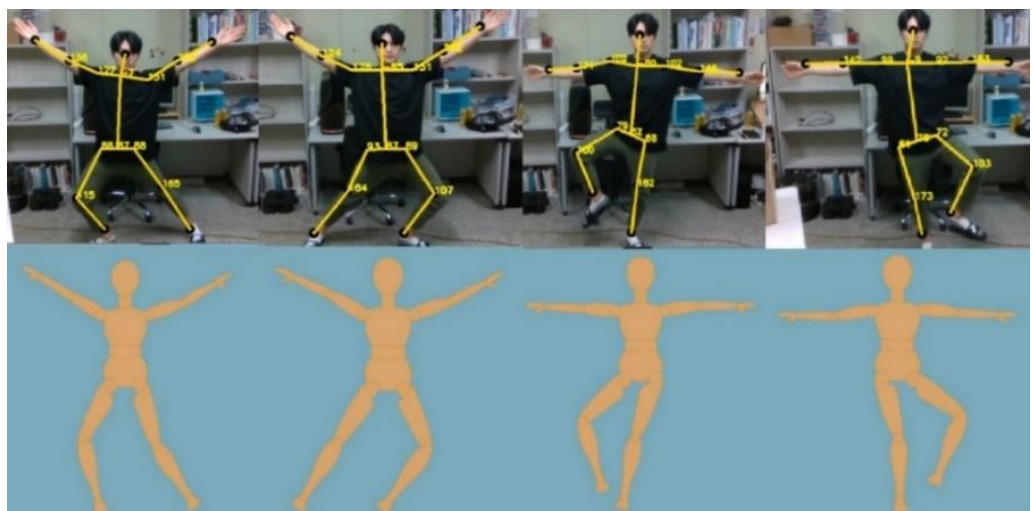

Figure 5. A puppet image produced in real motion and angle

By checking Table 1, we can see that the movement of the joints affects the angles on the data. In addition, it can be seen that data with varying joint variations has been stored for the opposite behavior. It can be seen that the resulting angle differs from the actual angle of thought. It should be noted that this is because 
it is the angle to the data, not the angle desired by the individual or physician. It should be noted that the angle of the joints shown in the table above is negative for opposite angles.

Table 1. Joint angle calculation table by motion

\begin{tabular}{lcccc}
\hline Pose & \multicolumn{2}{c}{ Warrior posture } & \multicolumn{2}{c}{ Holding one foot and balancing } \\
Direction & Right(Angle) & Left(Angle) & Right(Angle) & Left(Angle) \\
\hline Body & 1 & 2 & 5 & -7 \\
Left Arm & -33 & -34 & -7 & -6 \\
Right Arm & 20 & 20 & 3 & 5 \\
Left Leg & -36 & -34 & $\mathbf{1 2}$ & $\mathbf{- 5 0}$ \\
Right Leg & 28 & 29 & $\mathbf{5 8}$ & $\mathbf{- 1 6}$ \\
Left Knee & $\mathbf{- 3 2}$ & $\mathbf{3 5}$ & $\mathbf{2}$ & $\mathbf{- 1 5}$ \\
Right Knee & $\mathbf{3 6}$ & $\mathbf{- 3 4}$ & $\mathbf{- 2 3}$ & $\mathbf{5}$ \\
Left Elbow & -29 & -35 & 6 & 6 \\
Right Elbow & 31 & 33 & 1 & \\
\hline (Bold text identifies difference for opposite behavior) & & &
\end{tabular}

\section{CONCLUSION}

This paper confirmed the lack of muscle strength of the elderly, and made suggestions on exercise contents that could have practical effect on it. A method that can correct the accuracy of existing joint tracking algorithms in basic motion was presented, and the calculation of angles in the joint tracking was carried out. In addition, it was expanded and linked to the contents, and the actual test was conducted for the effective operation with the cooperation of the hospital to confirm the possibility. Looking back at the overall study, for basic joint tracking, tools such as Kinect can also be used to determine the angle of each joint. However, for other algorithms that do not, there is no way that angles cannot be determined. In addition, improved data computations allowed various activities of contents while increasing accuracy. Further research will help research and development into content that allows joint angles to actual expert movements to be stored and followed for correct movements to be compared with those used for testing.

\section{ACKNOWLEDGEMENTS}

This research was supported by the MSIT (Ministry of Science and ICT), Korea, under the Grand Information Technology Research Center support program (IITP-2020-2016-0-00318) supervised by the IITP (Institute for Information and communications Technology Planning and Evaluation) and Institute of Information and communications Technology Planning and Evaluation (IITP) grant funded by the Korea government (MSIT) (No.2020-0-01791,'Busan AI Grand ICT Research Center Support Project')

\section{REFERENCES}

[1] S. T. Hong, "Low Birth Rate, Rapid Aging, and Korean Society: Toward welfarism beyond growthism," Democratic Society end policy Studies, vol. 11, pp. 15-40, 2007.

[2] Y. S. Lee, "The Effects of Population Ageing to Regional Economy," Korea Planning Association, vol. 43, no. 7, pp. 7-10, Dec. 2008

[3] S. J. Min, “Aging Vulnerability Index of Korea," The Korean Journal of Economics, vol. 19, no. 1, pp. 49-80, Jun. 2012.

[4] S. J. Kim, "A Critical Study on the Population Policy of Korea in the Low Fertility and Aging Society," The Korean Urban Geographical Society, vol. 22, no. 2, pp. 143-158, 2019.

[5] S. E. Jung, Y. Okubo, Y. Osuka, S. Seino, J. S. Park, H. S. Nho and K. Tanaka, "Older Korean adults have lower physical function despite longer exercise times compared to their Japanese counterparts: A Japan-Korea comparative study," Geriatrics and Gerontology International, vol. 18, no. 4, pp. 576-583, Dec. 2017.

[6] N. Y. Ahn and K. J. Kim, "Effects of therapeutic exercise for stroke Patients," Korean Acadeamy of Kinesiology, vol. 10, no. 2, pp. 45-54, Oct. 2008.

[7] S. N. Nam, J. H. Kim and Y. S. Cho, "The Effect of Affected Side of Muscle Strength and Articular Moving Range of Persons With Cerebral Apoplexy Hemiplegia on Aqua-Rehabilitation," Korean Society Adapted Physical activity and Exercise, vol. 12, no. 2, pp. 79-88, Jun. 2004.

[8] S. H. An, H. J. Lee, W. S. Lim and H. S Lee, "The Effect of the Group Therapeutic Exercise Program on the Cognitive Function, ADL, and Balance-Performing Ability in Elderly Persons," Korean Research Society of Physical Therapy, vol. 13, no. 2, pp. 26-34, 2006.

[9] Y. Ho, "A study on the exercise effect on the obese and the necessity of group rehabilitation sprots," Korean Journal of Sports Science, vol. 20, no. 2, pp. 257-267, Apr. 2011.

[10] S. Keesara, A. Jonas and K. Schulman, "Covid-19 and Health Care's Digirtal Revolution," The New England Journal of Medicine, vol. 382, no. 23, Jun. 2020 
[11] Y. J. Lee, "The Effect of Information Conditions on Mental Health among Elderly," The society of Digital Policy \& Management, vol. 11, no. 10, pp. 17-19, 2013.

[12] Y. J. Shin and M. J. Gu, "Exploratory study on information education for the elderly and life satisfaction of the elderly," Interdisciplinary Journal of Adult and Continuing Education, vol. 13, no. 4, pp. 119-147, Nov. 2010.

[13] K. H. Paek, J. S. Bong and Y. T. Shin, "An Empirical Study on the Factors and Resolution Methods of the Smart Divide of Older Adults," Korean Institute of Information Scientists and Engineers, vol. 42, no. 10, pp. 1207-1221, Oct. 2015.

[14] D. G. Choi and J. W Jang., "A Study on the Develeopment of Exercise Contents Based on Deep Learning Joint Tracking for Improving the Strength of Old Age," The 2020 Summer Conference of Korea Institute of Information and Communication Engineering, vol. 24, no. 1, 2020, pp. 107-109.

[15] Z. Cao, G. Hidalgo Martinez, T. Simon, S. Wei and Y. A. Sheikh, "OpenPose: Realtime Multi-Person 2D Pose Estimation using Part Affinity Fields," IEEE Transactions on Pattern Analysis and Machine Intelligence, vol. 43, noo. 1, pp. 172-186, Jul. 2019, doi: 10.1109/TPAMI.2019.2929257.

[16] Shih-En Wei, et al., "Convolutional Pose Machines," arxiv.org/abs/1602.00134, 2016.

[17] Cao, Zhe, et al, "Realtime Multi-Person 2D Pose Estimation using Part Affinity Fields," Proceedings of the IEEE conference on computer vision and pattern recognition, 2017, pp. 7291-7299.

[18] S. P. Lloyd, "Least Squares Qeantization in PCM," IEEE Transactions on Information Theory, vol. 28, no. 2, pp. 129-137. 1982.

[19] J. A. Hartigan and M. A. Wong, “Algorithm AS 136: A K-Means Clustering Algorithm,” Journal of the Royal Statistical Society, vol. 28, no. 1, pp. 100-108, 1979.

[20] C. A. Gonano and R. E. Zich, "Cross product in N dimensions: The doublewedge product," arXiv:1408.5799, 2014.

[21] X. L. Tian, C. Yang, Y. Hu and C. Tian., "Vector cross product in n-dimensional vector space," Mathematical Physics, arXiv:1310.5197, Oct 2013.

[22] K. N. Yasen, et al., "Hand detectiuon and segmectation using smart path tracking fingers as features and expert system classifier," International Journal of Electrical and Computer Engineering (IJECE), vol. 9, no. 6, pp. 5277-5285, Dec. 2019.

[23] H. Ham, J. Wesley, and Hendra, "Computer vision based 3D reconstruction : A review," International Journal of Electrical and Computer Engineering (IJECE), vol. 9, no. 4, pp. 2394-2402, Aug. 2019.

[24] M. C. Lee , K. Inoue , M. Tashiro, and M. Cho, "Three-Dimensional Visualization Technique of Occluded Objects Using Integral Imaging with Plenoptic Camera," Journal of Information and Communication Convergence Engineering, vol. 15, no. 3, pp. 193-198, Sep. 2017.

[25] S. Y. Lee, G. S. Son, H. J. Jeon and M. H. Lee, "The Effects of Therapeutic Exercise on the Balance and Gait in Older Adults," The Korean Society of Physical Therapy, vol. 19, no. 2, pp. 1-10, Apr. 2007.

[26] L. H. Im and Y. S. Jee, "Chronic Diseases and Exercise," Korea Coaching Development Center, vol. 8, no. 4, pp. 195-207, Dec. 2006.

\section{BIOGRAPHIES OF AUTHORS}

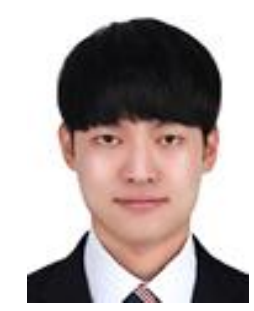

Donggyu Choi received the B.S. degree in Computer Engineering, and M.S. degree in Software Convergence from Dong-Eui University, Busan, Korea, in 2018 and 2020. Currently he is a researcher at the Computer Engineering Department, Institute of Smart IT, Dong-Eui University, Korea. Since 2020 he is perusing him Ph.D. degree from the Dong-Eui University, Korea. He Main research line concern include deep learning programming, autonomous driving, health care.

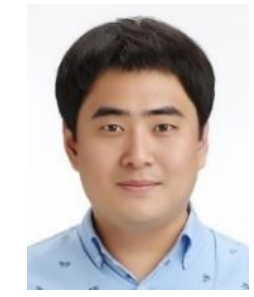

Minyoung Kim received the B.S. and M.S. and. Ph.D. degree in Computer Engineering from Dong-Eui University, Busan, Korea, in 2010 and 2013 and 2020. Since 2020 he has been assistant professor in the Department of Computer Engineering, Research Institute of ICT Fusion and Convergence, Dong-Eui University, Korea. He Main research line concern include Computer Network, autonomous driving, Vehicle Assist through IoT.

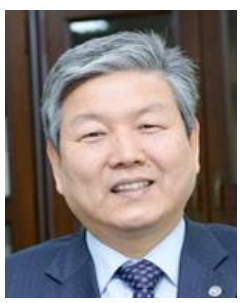

Jongwook Jang received the B.S. degree in Computer Statistics from Busan National University, Busan, Korea, in 1987, and M.S. degree in Computer Statistics from Chungnam National University, Daejeon, Korea, in 1991, and. Ph.D. degree in Computer Engineering from Busan National University, Busan, Korea, in 1995. Since 1995 he has been professor in the Department of Computer Engineering, Institute of Smart IT, Dong-Eui University, Korea. He Main research line concern Vehicle Network, Wire/Wireless Communication System. 